\title{
United States reviews safety of electroconvulsive therapy
}

$\mathrm{T}$ he United States government is taking a closer look at electroshock treatment, long a controversial treatment for severe depression and other psychiatric disorders, to determine if the therapy should be subject to more stringent oversight or reclassified as lower-risk.

After declining in use during the 1950s and 1960s, electroconvulsive therapy (ECT) has increased in popularity in recent years, with an estimated 100000 Americans receiving the treatment annually, most of them women. Most often, the treatment is used for severe depression, but it also is used for schizophrenia, catatonia and other conditions.

The question before the US Food and Drug Administration is whether to continue to place ECT equipment in Class III, the highest of three risk categories for medical devices, or shift it to a lesser-risk category. If ECT devices remain in Class III, manufacturers would be required to submit new information demonstrating their safety and effectiveness.

Supporters of electroshock treatment worry that manufacturers would have a hard time meeting such requirements and could be forced to pull the devices off the market; critics say it is long past time for ECT devices to be banned or at least be subjected to more rigorous safety testing and regulation.

Both sides turned out for two days of emotional hearings in January before a federal advisory committee. Those testifying in support of the treatment included Kitty Dukakis, the wife of former Democratic presidential candidate Michael Dukakis, who credited ECT treatments with helping to keep her alive. Critics, for their part, testified that the treatment destroys memories and leaves people brain-damaged.

While the advisory panel did not take a formal vote, a majority of committee members spoke in support of keeping ECT devices in Class III for the treatment of severe depression and a number of other conditions, accord-

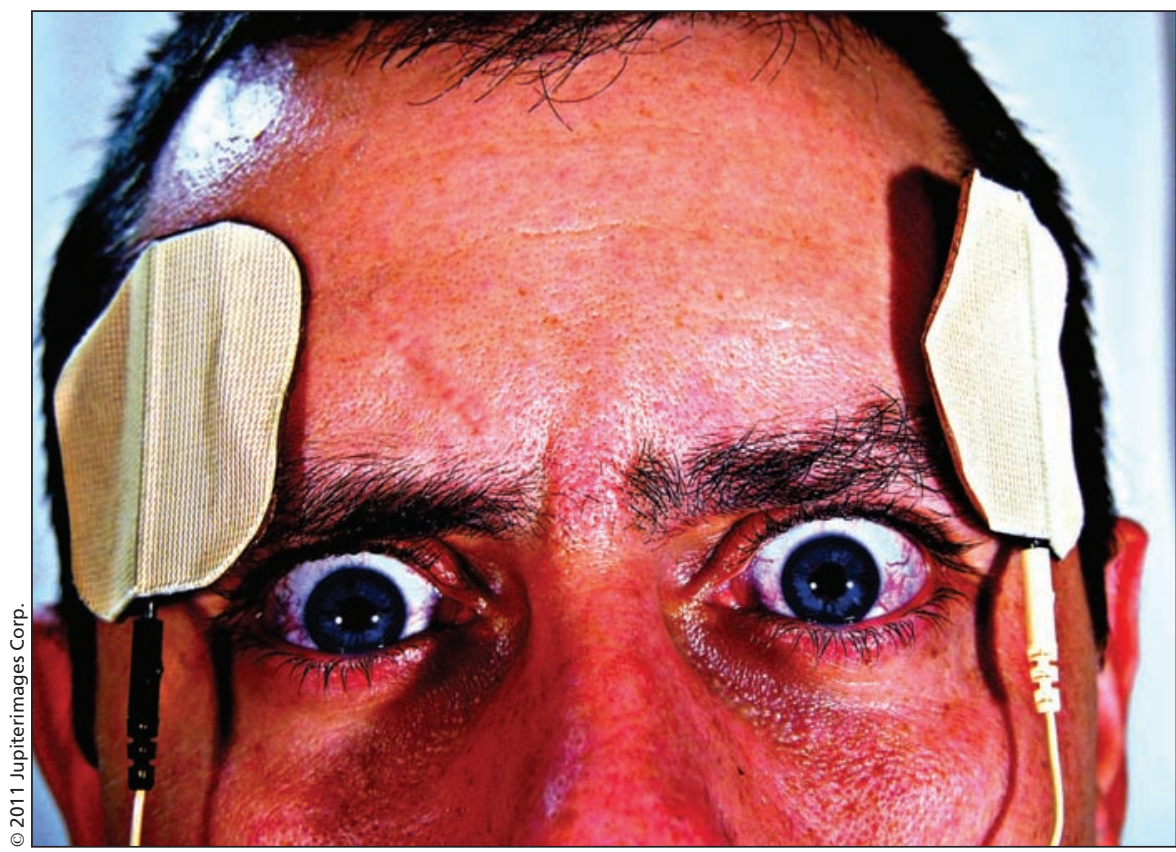

The Canadian Institute for Health Information has estimated electroconvulsive therapy has been administered to about 15000 Canadians annually since 2002 .

ing to FDA spokeswoman Karen Riley. The exception was catatonia, a condition for which there was no consensus on ECT classification, Riley says.

Now it's up to the FDA to decide how to classify ECT devices, which induce a seizure by applying electricity to the scalp. The agency typically follows the advice of its advisory committees, but is not required to do so. Riley says it could take a year or more for FDA to solicit public comment and issue a final rule. Manufacturers could have as long as 30 months after that to respond to any new requirements for safety and effectiveness information.

The FDA consideration of ECT devices is part of the agency's ongoing effort to review high-risk medical devices that were in use before the enactment of the Medical Devices Amendments of 1976, and were allowed to remain on the market without clearing a stringent review process. In 2009, the Government Accountability Office recommended that the FDA act expeditiously to ensure that grandfathered high-risk devices either meet stringent review standards or be reclassified as lower-risk.

Duke University Medical Center's Dr. Richard Weiner, speaking on behalf of the American Psychiatric Association, says the evidence that ECT is effective for major depressive disorder and other conditions is "highly compelling."

Weiner, a psychiatrist who has directed research on ECT and who testified at the hearings, adds that "it's well understood that there are risks, particularly in the area of memory function," which is why it's critical that patients give informed consent before undergoing the treatment. He stresses that the therapy is used sparingly, estimating that less than $5 \%$ of patients with major depressive episodes get ECT.

Weiner says that while ECT isn't a cure, it's an important treatment option "to bring somebody out of an acute episode" when they aren't responding to other therapies.

But John Breeding, a psychologist who helped found a Texas coalition against electroshock therapy, says it's unconscionable to subject people to 
ECT in the absence of rigorous testing to demonstrate that the treatment is safe and effective. He calls electroshock treatment a throwback to the days depicted in the movie One Flew Over the Cuckoo's Nest.

"A lot more people get hurt than helped," says Breeding, who testified at the hearings. "I consider it just a really horrible thing to do to people."

The National Alliance on Mental Illness, the largest organization of people with major psychiatric disorders, is recommending that ECT equipment be reclassified as Class II, or medium risk, to ensure it remains available as a treatment option.

"Overly burdensome restrictions on ECT devices would very likely make this treatment difficult to avail for those who need and want it the most," the organization said in a statement submitted to the FDA. It said that while the devices have not been through the rigorous research process typically required for high-risk devices, "in this instance, experience may be the best gauge for determining efficacy and safety."

The Canadian Institute for Health Information has estimated electroconvulsive therapy has been administered to about 15000 Canadians annually since 2002. It is increasingly being administered to Canadian seniors as a treatment for depression (www.cmaj.ca /cgi/doi/10.1503/cmaj.080360). Nancy Benac, Washington, DC

CMAJ 2011. DOI:10.1503/cmaj.109-3817 